\title{
Pyroptosis and Oral Cancer
}

\author{
Gargi Sarode ${ }^{1}$, Piyush Jain ${ }^{2}$, Namrata Sengupta ${ }^{3}$, Sachin Sarode ${ }^{4}$, Shankargouda Patil ${ }^{5}$ \\ The Journal of Contemporary Dental Practice (2019): 10.5005/jp-journals-10024-2682
}

Cell death is a basic biological occurrence which is required for the existence and growth of an organism. ${ }^{1}$ Programmed cell death sustains tissue homeostasis facilitating the elimination of redundant cells. $^{2}$ When an infectious disease sets in, cells that have been infected by pathogens prove harmful to the host eclls. ${ }^{2}$ Evidence has proved that cell death has a role in immune defense against infectious diseases. ${ }^{1}$ Cell death can be broadly classified based on its initiating events, such as apoptosis, pyroptosis and oncosis.

Pyroptosis is an exceedingly inflammatory type of programmed cell death associated commonly with intracellular microbial infection and also follows an antimicrobial response. It was first designated in 1992 in macrophages infected with Shigella flexneri. ${ }^{2}$ Though apoptosis and pyroptosis both lead to programmed cell death, they are diverse phenomena. The term "pyroptosis" was coined by Cookson and Brennan. ${ }^{3}$

In salmonella-infected cells, Cookson and Brennan ${ }^{3}$ observed and revealed stimulus evoking cell death through common effector pathways. The caspase- 1 dependence of this cytotoxicity differentiates it from other forms of accidental cell death. It is a proinflammatory program to eliminate potentially dangerous cells. Pyroptosis has been described as an unconventional way of confiscating redundant cells. It has been termed as the screaming, alarm-ringing pro-inflammatory death of potentially dangerous cells where the timely recruitment of other cells or their roles is essential.

They proposed the term "pyroptosis" originating from a Greek word "pyro", linking to fire, fever, or inflammation in the context of the role of caspase- 1 and IL-1 $\beta$ in fever and inflammation, and "ptosis" to signify a falling, to explain proinflammatory programmed cell death. ${ }^{2,3}$

Pyroptosis has only been explained in macrophages and dendritic cells with a few proofs of caspase- 1 activity in other cells. ${ }^{2}$ The phenomenon is controlled by a caspase-1-dependent/ independent phenomenon. Caspase-1-independent pyroptosis is carried out by human caspase-4, 5 or mouse caspase-11. All are identified by swelling of the cells and their positive expression for annexin $\mathrm{V}$. There is a loss of membrane potential of the mitochondria of pyroptotic cells. ${ }^{1}$ It results in breakage of the cellular membrane releasing all the contents of cytoplasm.

Caspase-1-dependent pyroptosis needs initiation of the recognized inflammasomes. ${ }^{1}$ Inflammasomes are high-molecularweight protein complexes triggered with numerous infectious pathogen or physiological stresses provoking a discharge of pro-inflammatory cytokines. These cytokines employ native immune cells for defense. In this pathway, inflammasome sensors are activated, such as NLRP1b, NLRP3 and NLRC4. They help in recruitment of the cysteine protease caspase 1 in a macromolecular complex. ${ }^{1}$ Caspase 1 splits gasdermin D and helps pro-IL-1 $\beta$ and 18 to mature, commencing pyroptosis. ${ }^{1} \mathrm{IL}-1 \beta$ is a powerful initiator of inflammatory vasodilation. IL-18 fosters interferon (IFN)- $\gamma$ assembly in helper T cells, NK cells, and cytotoxic T cells and promotes local inflammation. Caspase-1-independent pyroptosis necessitates \begin{tabular}{l}
\hline${ }^{1-4}$ Department of Oral Pathology and Microbiology, Dr DY Patil Dental \\
College and Hospital, Dr DY Patil Vidyapeeth, Pune, Maharashtra, India \\
${ }^{5}$ Department of Maxillofacial Surgery and Diagnostic Sciences, Division \\
of Oral Pathology, College of Dentistry, Jazan University, Kingdom of \\
Saudi Arabia
\end{tabular}

Corresponding Author: Gargi Sarode, Department of Oral Pathology and Microbiology, Dr DY Patil Dental College and Hospital, Dr DY Patil Vidyapeeth, Pune, Maharashtra, India, Phone: +91 9823871462, e-mail: gargi14@gmail.com

How to cite this article: Sarode G, Jain P, et al. Pyroptosis and Oral Cancer. J Contemp Dent Pract 2019;20(9):1001-1002.

Source of support: Nil

Conflict of interest: None

stimulation of the nonrecognized inflammasome ${ }^{1}$ (Flowchart 1). Pyroptosis plays a twin role in tumor pathogenesis. On the one hand, the inflammatory mediators produced during pyroptosis are vital for the process of tumorigenesis and also has a role in drug resistance to chemotherapeutic drugs. Contrary to this, being a type of death, pyroptosis prevents the formation and progression of neoplasms. ${ }^{4}$

NLRP3 inflammasome was studied in 20 biopsied cases of oral squamous cell carcinoma (OSCC) including the malignant tumor and the adjacent non-pathological tissues. It was revealed that the expression levels of NLPR3 inflammasome associated genes (ASC, casp-1, IL- $\beta$ and NLRP3) were higher in tumor tissue. These levels are related to clinical and pathological characteristics of OSCCs. The study also stated an in vitro experiment where they concluded that ASC (apoptosis-associated speck-like protein) facilitates migration and invasion of OSCC cell lines, promoting metastasis. ${ }^{5}$ A similar finding was noted in hepatocellular cancer (HCC). Wei et al. discovered downregulated or absence of NLRP3 expression in HCC tissues. The manifestation was indirectly proportional to the pathological grade as well as clinical stage of HCC. This is an indicator that the NLRP3 inflammasome has a pivotal role in HCC progression. ${ }^{6}$

Recent studies have shown that administration of anthocyanin (a flavonoid) activates pyroptosis in OSCC with augmented NLRP3, caspase-1, and IL-1 $\beta$ expression. Gasdermin D (GSDMD), a pyroptosis-associated factor, was also increased with stimulation of the caspase- 1 inflammatory pathway. ${ }^{6}$ Thus, anthocyanin upregulates GSDMD, consequently contributing to the initiation of pyroptosis. ${ }^{7}$ Other studies have shown that flavonoids inhibit proliferation of OSCC cells, cell migration, and invasion through the arrest of $\mathrm{G} 1$ phase and mitochondria-mediated apoptosis. ${ }^{8}$

Data indicate that in OSCC, pyroptosis is stimulated after anthocyanin administration triggering loss of neoplastic cells inhibiting their migration and invasion. The effects of anthocyanin on OSCC cells were suppressed by the caspase-1 inhibitor AC-YVADCMK. ${ }^{7}$ Hence, anthocyanin stimulates destruction of OSCC cells by triggering pyroptosis and thus prevents development of cancer. 


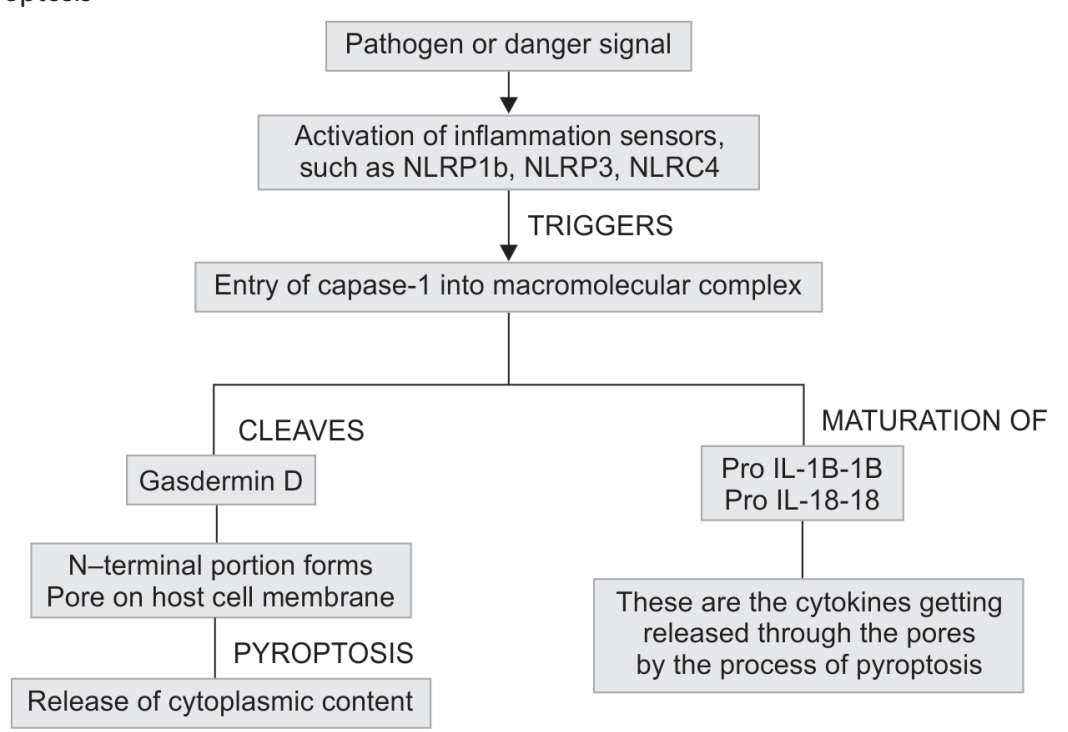

Furthermore, detailed studies are required to conclude the mechanism of pyroptosis and the role of anthocyanin to induce pyroptosis in OSCC cells to prevent its invasion and metastasis. Future studies indicated will be helpful in generating various therapeutic strategies applying or stimulating pyroptosis.

The recently established tumor pyroptosis treatment confirms prodigious prospective. Recent data show that chemotherapeutic preparations, miRNA, and similar treatment strategies can stimulate tumor pyroptosis, thus deterring the malignant development. There are many chemotherapeutic drugs, which can induce pyroptosis in tumor tissues. Strategies to downregulation of GSDMD should be the area of research. Suitable chemotherapeutic medications can be designated and applied as per the level of GSDME expression. The levels are upregulated in neoplastic cells improving sensitivity to chemotherapeutic medicines and diminishing resistance to drugs. ${ }^{4}$

Moreover, other proteins from gasdermin family should be vigorously explored to deliver new treatment strategies in OSCC. More investigations, experimentations, and clinical trials are desirable to investigate the conceivable use of the treatment strategy centered on pyroptosis for oral cancer. The phenomenon can also be exploited to understand the pathogenesis, progression, and invasion of OSCC and other disorders like potentially malignant oral disorders along with their treatment options.

As cumulative proof shows that pyroptosis has a double role of antitumor and tumor promotion in tumorigenesis, it will definitely have an enormous influence on the diagnosis and treatment of OSCC. However, current studies are preclinical in nature and are mostly performed on cell lines. To better understand the actual role of pyroptosis in OSCC it is important to conduct studies on human tissues.

In contrast to other malignancies of the body, OSCC is highly unique in terms of the degree of heterogeneity. ${ }^{9,10}$ Two important reasons associated with it are close association of microbes with cancer cells and premalignant state. Virtually every OSCC is secondarily infected and thus has a strong association with microbiota-associated modulation of tumor biology. Hence, there is a strong possibility of pyroptosis-associated alterations in the signaling pathways of OSCC. The second aspect is the association of OSCC with oral potentially malignant disorders (OPMDs). There is a plethora of such lesions, which could eventually transformed into OSCC. ${ }^{11,12}$
Some of the common OPMDs are leukoplakia, erythroplakia, lichen planus, and oral submucous fibrosis. It would be very interesting to study the role of pyroptosis in various OPMDs, which would help in designing future diagnostic, prognostic, and therapeutic strategies.

\section{References}

1. Man SM, Karki R, et al. Molecular mechanisms and functions of pyroptosis, inflammatory caspases and inflammasomes in infectious diseases. Immunol Rev 2017;277(1):61-75. DOI: 10.1111/imr.12534.

2. Miao EA, Rajan JV, et al. Caspase-1-induced pyroptotic cell death. Immunol Rev 2011;243(1):206-214. DOI: 10.1111/j.1600065X.2011.01044.x.

3. Cookson BT, Brennan MA. Pro-inflammatory programmed cell death. Trends Microbiol 2001;9:113-114. DOI: 10.1016/S0966842X(00)01936-3.

4. Xia X, Wang $X$, et al. The role of pyroptosis in cancer: pro-cancer or pro-"host"? Cell Death Dis 2019;10(9):1-3. DOI: 10.1038/s41419-0191883-8.

5. Wu CS, Chang KP, et al. ASC contributes to metastasis of oral cavity squamous cell carcinoma. Oncotarget 2016;7(31):50074-50085. DOI: 10.18632/oncotarget.10317.

6. Wei Q, Mu K, et al. Deregulation of the NLRP3 inflammasome in hepatic parenchymal cells during liver cancer progression. Lab Invest 2014;94:52-62. DOI: 10.1038/labinvest.2013.126.

7. Yue E, Tuguzbaeva G, et al. Anthocyanin is involved in the activation of pyroptosis in oral squamous cell carcinoma. Phytomedicine 2019;56:286-294. DOI: 10.1016/j.phymed.2018.09.223.

8. Iriti M, Varoni EM. Chemopreventive Potential of Flavonoids in Oral Squamous Cell Carcinoma in Human Studies. Nutrients 2013;5(7):2564-2576. DOI: 10.3390/nu5072564.

9. Sarode G, Sarode SC, et al. Is oral squamous cell carcinoma unique in terms of intra-and inter-tumoral heterogeneity? Trans Res Oral Oncol 2017;2:11-16. DOI 10.1177/2057178X17703578.

10. Nilendu P, Sarode SC, et al. Mutual concessions and compromises between stromal cells and cancer cells: driving tumor development and drug resistance. Cell Oncol (Dordr) 2018;41(4):353-367. DOI: 10.1007/s13402-018-0388-2.

11. Sarode SC, Sarode GS, et al. A new classification for potentially malignant disorders of the oral cavity. Oral Oncol 2011;47(9):920-921. DOI: 10.1016/j.oraloncology.2011.06.005.

12. Sarode SC, Sarode GS, et al. Oral potentially malignant disorders: precising the definition. Oral Oncol 2012;9(48):759-760. DOI: 10.1016/ j.oraloncology.2012.02.025. 